\title{
AOF - first on-sky performance of the GALACSI GLAO mode (or how to close 10 loops in less than 5 minutes)
}

Johann Kolb $^{b^{*}}$, Pierre-Yves Madec ${ }^{\mathrm{a}}$, Robin Arsenault ${ }^{\mathrm{a}}$, Sylvain Obertia , Jérôme Paufique ${ }^{\mathrm{a}}$, Paolo La Penna $^{a}$, Stefan Ströbele ${ }^{a}$, Robert Donaldson ${ }^{\mathrm{a}}$, Christian Soenke ${ }^{\mathrm{a}}$, Marcos Suárez Valles ${ }^{\mathrm{a}}$, Mario Kiekebusch $^{\mathrm{a}}$, Javier Argomedo ${ }^{\mathrm{a}}$, Miska Le Louarn ${ }^{\mathrm{a}}$, Elise Vernet ${ }^{\mathrm{a}}$, Pierre Haguenauer ${ }^{\mathrm{b}}$, Philippe Duhoux $^{\mathrm{b}}$, Emmanuel Aller-Carpentier ${ }^{\mathrm{a}}$, Jose Javier Valenzuela ${ }^{\mathrm{b}}$, Juan Carlos Guerra ${ }^{\mathrm{b}}$

aEuropean Southern Observatory, Karl-Schwarzschild-Str. 2, 85748 Garching bei München, Germany

${ }^{\text {b} E u r o p e a n ~ S o u t h e r n ~ O b s e r v a t o r y, ~ A l o n s o ~ d e ~ C o ́ r d o v a ~ 3107, ~ V i t a c u r a, ~ C a s i l l a ~ 19001, ~ S a n t i a g o ~ d e ~}$ Chile, Chile

\begin{abstract}
GALACSI is an AO module that equips the VLT Yepun, making use of the recently installed Deformable Secondary Mirror and 4 Laser Guide Star Facility. In its Wide Field Mode, it feeds the MUSE instrument with a GLAO corrected FoV in the visible of 1 arcmin square.

This paper will present the results of the first commissionings that took place from March to June 2017: Acquisition and Observation, taking over the telescope Active control and AO performance vs. Turbulence conditions.
\end{abstract}

Keywords: AO Facility, AOF, VLT, GLAO, commissioning, GALACSI, DSM, 4LGSF, MUSE

\section{INTRODUCTION}

The Adaptive Optics Facility (AOF) is an upgrade of the VLT Yepun 8-meter diameter telescope that includes several components. The M2 Dornier is replaced by a new M2 Unit ${ }^{[1]}$ hosting:

- The DSM (1172 actuators controlled at $1 \mathrm{kHz}$ ) allowing to perform Field Stabilization (+/- 3.3 arcsec on-sky), Chopping (5.5 arcsec on-sky) and turbulence correction

- The Hexapod, allowing to position the DSM in focus and centering, with the same range as the M2 Dornier It also includes four Laser Guide Star Units ${ }^{[2]}$, each of them including:

- A laser unit delivering22 W@589 nm

- A launch telescope delivering a 22-cm beam @ $\mathrm{e}^{-2}$

- A Field Steering Mirror (FSM) allowing to position the laser as far as 7.6 arcmin from the telescope optical axis

- A Jitter Mirror allowing to compensate for uplink and downlink laser jitter: +/- 13 arcsec on-sky at $1 \mathrm{kHz}$. To be noted: GALACSI is not using the 4LGSF JMs

- A Beam Expander Unit allowing to focus the laser into the $\mathrm{Na}$ layer

GALACSI $^{[3]}$ is bolted onto the Nasmyth B Adaptor/Rotator. It serves MUSE and features in its Wide-Field Mode:

- A Laser Guide Star WFS path including a Focus Compensator that adjusts the focus of the 4 LGS on the 4 Shack Hartmann WFSs (40x40 sub-apertures, 5 arcsec FoV/sub-aperture, $1 \mathrm{kHz}, 1$ electron Read-out Noise) and 4 Jitter Mirrors (one per WFS) to correct for laser jitter (+/-3 $\operatorname{arcsec}$ on-sky at $1 \mathrm{kHz})$ 
- A Natural Guide Star Tip-Tilt Sensor path including a Field Selector to select the tip-tilt star within the GALACSI patrol field, and the tip-tilt sensor itself (1 sub-aperture, 2.6 arcsec FoV, $200 \mathrm{~Hz}, 1$ electron Read-out Noise)

- A Commissioning Camera, fed by a retractable folding mirror

GALACSI can also be configured in a Narrow-Field Mode for LTAO correction using an infrared 2x2 SHWFS for Tiptilt and Focus correction on the on-axis Scientific object itself ${ }^{[4]}$.

GALACSI has been intensively tested in the ESO laboratories in Garching, on the test bench ASSIST that allowed simulating LGS and NGS, atmosphere and the VLT optics including the DSM ${ }^{[5]}$. The commissioning of the GALACSI Wide-Field Mode will be completed in 3Q2017, the one of GRAAL in 4Q2017 and GALACSI NFM in 2Q2018.

\section{AOF CONTROL}

The Control of the non-adaptive VLT relies on the Sensor Arm which is hosting a Guide Probe (tip-tilt sensor) and a Shack-Hartmann (Active Optics WFS):

- To perform Field Stabilization (FS) the Tip-tilt commands are sent to M2 with a control frequency up to $65 \mathrm{~Hz}$ (fast mode of FS). Tip-tilt is offloaded from M2 to the telescope axes at $1 \mathrm{~Hz}$ with a low gain (0.02).

- To control the Active Optics, the Shack Hartmann measurements are projected onto the first 29 elastic modes of M1; Focus and Coma are sent to M2 positioning actuators while the remaining modes are sent to M1 at a control period between 30 and 45 seconds (depending on the guide star brightness)

During AOF operations, the telescope SH WFS cannot be used anymore to control the active optics because:

- It sees the corrections applied by the DSM

- The DSM is controlled at $1 \mathrm{kHz}$ (Primary loops, see §2.1) while the SH is delivering a measurement every $45 \mathrm{~s}$

- All M1 deformations and DSM motions are corrected by the High Order loop

Some of the AOF auxiliary loops (see $\$ 2.2$ ) have thus been put in place to take over those telescope functions. A schematic of the primary and auxiliary loops is provided on Figure 1.

Finally a plethora of bootstrap, secondary and monitoring loops complete the control scheme of the AOF (see §2.3).

\subsection{Primary (fast) loops}

\section{Tip-tilt loop:}

- A Natural Guide Star is selected in a ring between 50 and 110 arcsec from the field center

- Tip-tilt measurements are elaborated applying a Weighted Center of Gravity algorithm to the NGS image

- Delta tip-tilt commands are computed from the tip-tilt measurements

- An integrator is applied to the tip-tilt delta commands resulting in a tip-tilt command

- The tip-tilt command is multiplied by a tip-tilt vector, to get a tip-tilt control vector defined in the DSM zonal control space

- Control frequency: $200 \mathrm{~Hz}$

\section{High Order loop:}

- For each LGS WFS, slopes are computed using a classical Center of Gravity algorithm. Each slope vector is multiplied by a control matrix to deliver a DSM delta-control vector. Tip-tilt (i.e. laser jitter) is filtered out from the control matrix.

- The 4 DSM delta-control vectors are averaged 
- An integrator is applied to the final DSM delta-control vector resulting in a High Order control vector

- The tip-tilt control vector is added to the High Order control vector to get a final DSM control vector

- Control frequency: $1 \mathrm{kHz}$

Jitter Loop: the purpose of this loop is to center and stabilize the image of the LGS into the 4 LGS WFS sub-apertures. For each laser path:

- The LGS WFS slope vector is projected into delta Jitter commands

- A PI (Proportional-Integral) is applied to the delta Jitter commands resulting in a Jitter command

- The Jitter command is applied to the LGS WFS Jitter mirror

- Control frequency: $1 \mathrm{kHz}$

- The Jitter commands are offloaded to the LGSU Field Steering Mirror when their time-average $(1 \mathrm{~s}$ window) is larger than a pre-defined value.

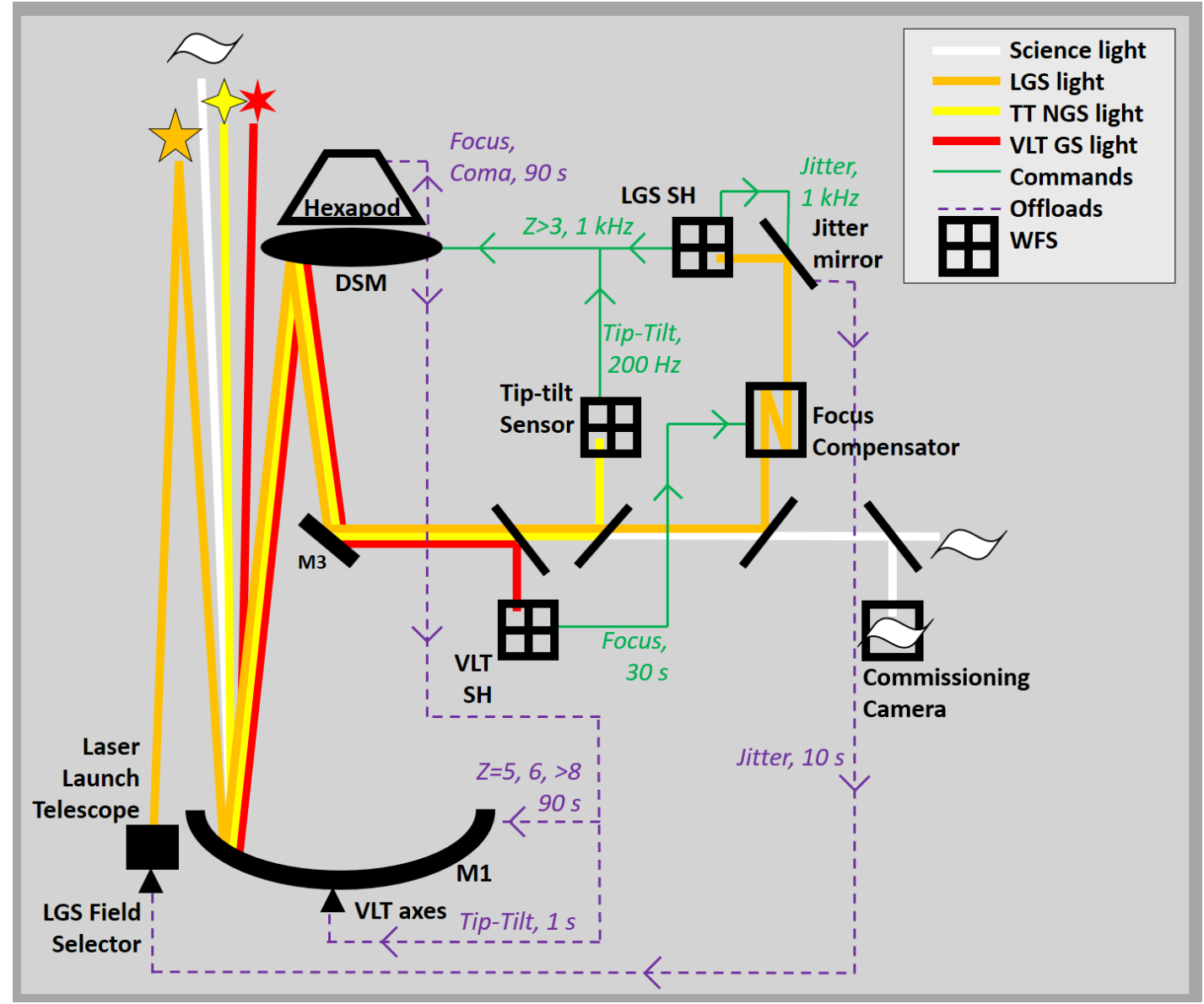

Figure 1 Control scheme of the AOF using GALACSI in Wide-Field Mode.

\subsection{Auxiliary (slow) loops / offloads}

Active optics is now controlled making use of the long-term shape of the DSM:

- DSM commands are averaged over $30 \mathrm{~s}$ 
- The average command is projected onto the first 29 elastic modes of M1 (via a Control Matrix calibrated on sky)

- Focus and Coma are sent to the DSM hexapod

- The other modes are sent to M1

- Control frequency: every $90 \mathrm{~s}$

DSM tip-tilt offloaded to the telescope axis as it was done with the Dornier (frequency $1 \mathrm{~Hz}$ - gain 0.02 , same interface)

The focus of MUSE (as for any other VLT instrument) is defined by the active optics SH WFS. In AOF operations, the atmospheric focus is corrected from the LGS WFS so variation of the Na layer altitude impacts on the quality of the instrument focus. To overcome this problem, the active optics SH WFS is used as a Truth Sensor: the residual defocus it measures is used to offset the Focus Compensator at a control period of $90 \mathrm{~s}$.

\subsection{Secondary loops, bootstrap and monitoring.}

We call secondary loops all the processes that either allow bootstrapping the starting reference state of the system or that run in the background to ensure a continuous stability, robustness and performance during an observation, as well as their monitoring.

4LGSF pointing model: Because of gravity and flexures, the pointing of the Laser Launch Telescopes must be adjusted by a simple model (including also the static alignment error of the WFSs w.r.t. the nominal position) ensuring that the LGS spots on sky will be within the capture range of a spiral search (see §3).

LGS Dark Follower: Every minute, evaluate the offset of the dark of the LGS WFS cameras:

- Take measurements of the dark outside the pupil in each channel of the detector (out of eight)

- If the offsets are larger than a pre-defined threshold, correct the dark map of the detector and apply it

LGS low flux freezing: It makes the HO loop extremely robust to clouds and aircraft alarms:

- When the flux in one sub-aperture of an LGS WFS is lower than a pre-defined threshold, the associated measurement is set to $[0,0]$.

- When more than a given number of sub-apertures have a too low flux, all the measurements of this WFS are set to 0 and therefore the delta DSM commands are equal to 0. If all WFSs are in this situation the DSM is effectively frozen.

- As soon as the flux is back, the WFS delivers again measurements, and the DSM starts correcting again.

- This check is made at the loop rate: $1 \mathrm{kHz}$

LGS WFS weighting map update: the purpose of this loop was to measure the LGS spots size (elongated and nonelongated dimensions) and based on that, generate weighting maps (after multiplication of the FWHMs by a weighting factor) to be updated every 5 minutes to cope with changing seeing condition in the case of a low return flux. It turned out that the return flux from the 4LGSF is in the bright-end regime in all observed conditions so that we decided to stick to a simple Center of Gravity algorithm with threshold at 3 sigma of the noise. The spot measurement part is however still running to provide estimations of LGS spot size and sodium layer thickness.

LGS focalization: The measured LGS spot size described above can be minimized by controlling the Beam Expander Unit inside the 4LGSF Launch Telescopes, but it turned out during commissioning that the spot size is little sensitive to this focalization and that the focusing model is good enough, so this function is not used.

WFS/DSM mis-registration: It can evolve with parameters like telescope elevation, and when the shift reaches more than $20 \%$ of a sub-aperture, performance drop if the control matrices are not updated. Mis-registrations have been measured during the commissioning to be repeatable and linear with elevation (see Figure 2). The strategy is then to pre-compute control matrices for 3 different elevation angles, and apply them depending on the telescope position (during preset and during observation if necessary). 
To allow measuring mis-registration, we have developed a sophisticated method delivering results every 10 minutes in closed loop during observation. This method has been tested in the laboratory and the whole sequence was validated on sky, but as a Look-up Table is sufficient we are not using this method in GALACSI WFM. The mis-registrations are however estimated and logged for trending.

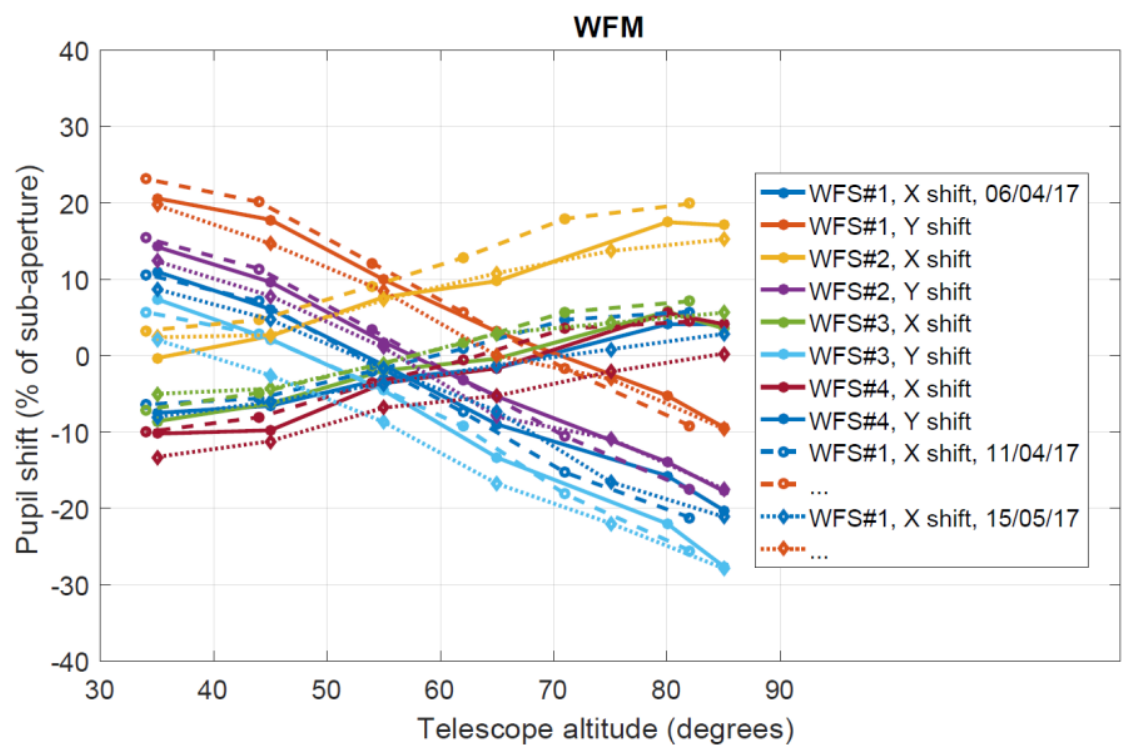

Figure 2 Pupil shift between the DSM and the $X$ and $Y$ axes of each of the 4 LGS WFSs (curves with 8 different colors), measured on sky at different dates ( 3 lines styles).

Focus control: For a given sodium layer altitude, the distance to the layer changes with elevation. When the telescope is tracking, the Focus Compensator is moving to compensate for the change in sodium layer distance. Its control is based on a Feedforward, and offsets are applied depending on focus measurements delivered by the Truth Sensor (see §2.2).

At preset, the altitude of the sodium layer is not known so before closing the HO loop, the focus term is measured on the WFSs, averaged and minimized by offsetting the Focus Compensator so that when closing the loop, no focus will be added to the one properly set by the first two cycles of Active Optics.

Tip-tilt Control Matrix de-rotation: During observations, there is a differential rotation between the tip-tilt sensor coordinate system and the DSM one. To compensate for this rotation the tip-tilt control matrix is de-rotated every two minutes. The de-rotation angle is computed using an analytical law; coefficients of this model have been calibrated using the GALACSI Calibration Unit.

NGS WFS weighting map update: Tip tilt measurements are computed using a Weighted Center of Gravity to better deal with faint stars. When the seeing changes, weighting maps need to be updated:

- The loop first closes with a default weighting map to capture the NGS. After 2 seconds of stabilization, the FWHM of the tip-tilt star image is measured on an average image (50 frames only, to quickly get a valid map)

- If the measured FWHM passes sanity checks, a new weighting map is generated numerically using a Gaussian model (and a weighting factor of 2.5 times the measured FWHM) then loaded into the RTC (without opening the loops)

- The process is then immediately repeated with a longer averaging (600 frames), and then again every 10 minutes.

NGS WFS Background follower: Every 5 minutes, we evaluate the offset of the background of the tip-tilt sensor camera:

- Take measurements of the background inside the 32x32 pixels real time window but outside the central $16 \times 16$ pixels window used for centroiding 
- If the offsets are larger than a pre-defined threshold, correct the background map of the detector and apply it

This secondary loop avoids recording on-sky background at preset. It saves time during the acquisition sequence and make it simpler, i.e. more robust. The fitted Gaussian also provides spot size and flux estimates that can be logged and monitored.

NGS low flux alarm: The purpose of this alarm is to detect when there is not enough flux in the image of the tip-tilt star and to open the loop. It cannot be based on the instantaneous measurement of the flux because at the GALACSI WFM limiting magnitude of 18.5 the flux of a few tens of ADUs spread over several pixels (especially in worse seeing conditions) is drowned in the background noise and cannot thus be estimated accurately. The strategy is the following:

- Compute the rms of the $\mathrm{X}$ and Y residual gradients (measured slope in pixels) of the visible tip-tilt camera over a $1 \mathrm{~s}$ window.

- Check if any of the two rms value is higher than 0.35 (pixels):

If no, wait for the rms value of the following 1s window

- If yes, average 25 images and sum the values of the four center pixels. If the sum is below a threshold (100 ADUs), open the TT loop (to avoid drifts) and warn the operator that there is probably no bright enough star in the TT guiding camera, for him to take the decision on continuing the observation or not.

M1 passive support: When the telescope is tracking, M1 is moving in its cell (lateral shift and tip-tilt). The active optics loop of the TCS sends a re-centering command to M1 at the same time it sends the shaping command. If the decentering is larger than a pre-defined value (so-called dead-band), M1 is re-centered into its cell. As in AOF operations the telescope Active Optics loop is disabled, the AOF control had to replicate this function: together with the offload of the DSM onto M1 and the M2 hexapod, the TCS re-centering command is sent to M1 with the same dead-band parameters.

DSM skipping alarm: The particular technology of the DSM makes that its commands are not limited in stroke (like a piezo-electric Deformable Mirror) but in the force applied to each electrode. Thus at each loop cycle, the commands sent by the Real-Time Computer are analyzed within the DSM and if at least one electrode or one mode exceeds the allocated force, the command is not applied and an echo signal informs the RTC. Because the DSM was specified to correct most turbulent conditions encountered in Paranal without reaching the limit force on any actuator (frame skipping occurs very rarely), this brutal strategy was chosen for its simplicity over a more complex one of re-computing the discarded command to minimize forces.

However, the DSM skipping for more than 1000 consecutive frames means that there is a problem, it triggers an alarm and warns the operator.

Atmospheric Monitor: From AO real-time data (4 LGS WFSs slopes and DSM commands) some turbulence and performance parameters are computed every two minutes like the seeing, outer scale and ratio of corrected variance to the total.

\section{AOF ACQUISITION SEQUENCE}

The Acquisition sequence of the AOF (in that case of GALACSI WFM) encompasses all the steps from sending a preset to the telescope to the point where all the Loops and Offloads are closed and the observation with the Science instrument can begin. The overhead brought using the AOF with respect to the non-adaptive telescope starts when this one would be ready to start scientific observations, i.e. after two cycles of its Active Optics loop.

The successive phases of the GALACSI WFM acquisition sequence are as follows:

- $\quad$ Preset phase. In parallel: 
- The 4LGSF Field Steering Mirrors are sent to point to the GALACSI WFM configuration (slow motion that can continue in parallel to the telescope slewing) and the Beam Expander Units to focalize the LSG beams on the sodium layer at the altitude where the telescope will be preset.

- The telescope is preset to the desired coordinates

O The RTC is configured to WFM and its link to the DSM is enabled

- All GALACSI motors are sent to their reference or pre-computed position

- The read-out of all the WFS detectors starts in a safe mode (no amplification gain. At the same time, the monitoring of the DSM skipping starts (for the alarm)

At that point, the sequence is waiting for the first cycle of Telescope Active Optics correction (i.e. relatively good optical quality) to proceed further.

\section{- $\quad$ Tip/Tilt Sensor Bootstrap}

$\circ$ Based on the input NGS magnitude, the expected filter and camera gain settings are computed. The filter is then put in place. An average image is recorded of the $50 \operatorname{arcsec}^{2}$ acquisition FoV and if no risk of over-illumination is detected, the computed amplification gain is finally set (only 3 combinations possible: 1,10 or 100 ).

- An average image of the $50 \operatorname{arcsec}^{2}$ acquisition FoV is taken and a pop-up display panel prompts the user to confirm or select the position of the NGS, which may lead to a NGS Field Selector offset to center the star in the $32 \times 32$ pixels tracking window.

Usually around that point the Telescope Active Optics correction completes its second cycle so the AOF overhead starts.

- LGS Acquisition

- The flux is measured on the LGS WFSs and if no risk of over-illumination is detected, the nominal amplification gain of 100 is set.

- The control of the Jitter and field Steering Mirror in the 4LGSF Launch Telescopes is passed to SPARTA.

- A spiral search is performed for all 4 WFSs in parallel by sending a search pattern as disturbance to the 4LGSF Jitter Mirror actuators (range $+/-13$ arcsec) and measuring simultaneously the flux in the WFSs.

- At the end of the search the barycenter of the flux vs. Jitter Mirror position is computed for each WFS and the FSM actuators sent at that position.

- In the case of GALACSI the Jitter control is then switched from the 4LGSF actuators to the GALACSI internal ones

- The flux in the WFSs is checked against a threshold (i.e. the LGS is in their FoV)

$\circ$ The average focus on the 4 WFSs is minimized by offsetting the Focus Compensator

- High-order Loop closure

- The Jitter loop and its offload are closed to stabilize the LGS spots in the WFSs

- The High-Order Control Matrix is loaded from the Look-up Table as function of telescope altitude

- The Field Stabilization of the Telescope is stopped and the "Probe Guiding Mode" is engaged, i.e. the guide probe keeps on following its guide star (and the offload loop of the DSM Tip-tilt to the telescope axes continues). This is required to keep the image of the guide star at the center of the active optics Shack-Hartmann, which has to deliver an accurate focus measurement during observation (Truth Sensor).

- The communication channel from the telescope to the DSM (Field Stabilization) is disabled 
- The Telescope Active Optics is stopped

○ The high-order loop is closed, and automatically together with it the secondary loops of Misregistration estimation, spot size measurement, Dark follower and Atmospheric monitor. At the same time, the monitoring of the sub-apertures under-illumination starts (for the low-flux command freezing and alarm).

- $\quad$ NGS Loop Closure

- The Tip-Tilt sensor detector is set to tracking mode (32x32 pixels read out at $200 \mathrm{~Hz})$

- The Tip-Tilt loop is closed, and automatically together with it the secondary loops of weighting map update and background follower. At the same time, the monitoring of the low flux starts (for the alarm).

- Auxiliary loops closure

- The Active Telescope Control loop is closed (period of 90 seconds). It synchronizes the DSM offload to the telescope, the Focus Truth sensor loop and the M1 Passive Support re-centering.

- The High-Order Control Matrix update is engaged, based on telescope altitude checked every 60 seconds

- The Tip-Tilt Control Matrix de-rotation is engaged, based on the Adaptor/Rotator position checked every 120 seconds.

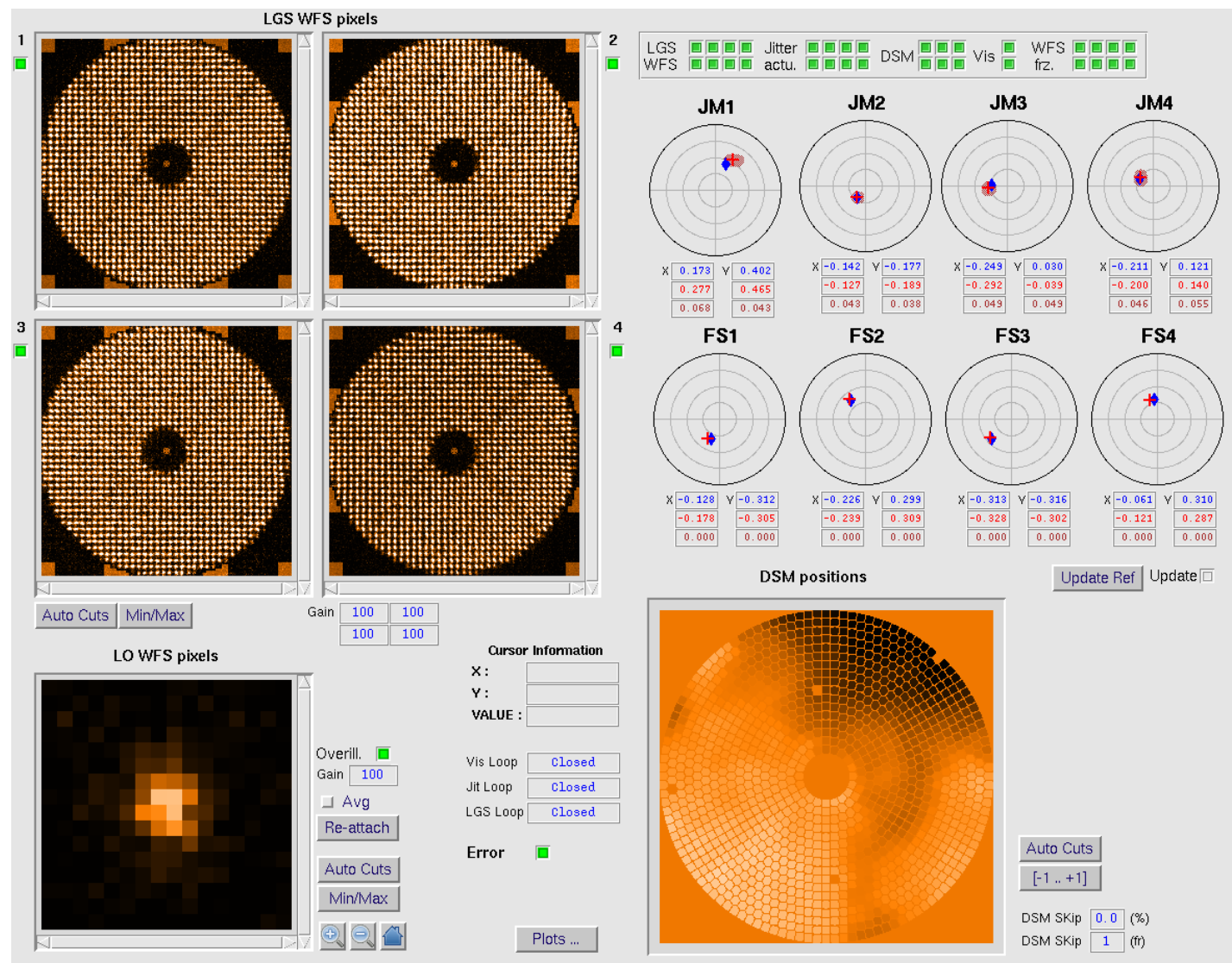

Figure 3 AOF Display panel. Top-left: the 4 SH WFSs. Bottom-left: the TT WFS. Bottom-right: DSM commands. Top-right: the 4 Jitter Mirror Actuators and Steering Mirrors commands. Bottom-center: status of the primary loops and detectors amplification gain. 
This Acquisition sequence is fully automated, requires only once an interaction with the operator (to select/confirm the NGS position) and adds an overhead of less than a minute (we usually measure 50 seconds) to the operation of the Telescope without the AOF. During observations, the operator can monitor the sensors and actuators of the AOF in a single panel, as shown on Figure 3.

\section{GLAO PERFORMANCE ON SKY}

We have used three commissioning runs from March to April 2017 to optimize the acquisition (see $\S 3$ ) and control (see $\S 2$ ) of GALACSI WFM, and to measure its performance in various turbulence conditions. Two additional runs were used to couple the AO system with its instrument MUSE.

As GALACSI feeds an Integral Field Spectrograph, the requirement on its performance was to improve by a factor of 2 the "Ensquared" Energy (EE) at $750 \mathrm{~nm}$ in a spaxel of MUSE of size $0.2 \operatorname{arcsec}^{2}$, under seeing conditions of 1.1 arcsec. This performance has been verified in the laboratory ${ }^{[5]}$ and on sky using the on-board Commissioning Camera (Comcam) in GALACSI, a Visible Technical CCD equipped with optical filters, and having a plate scale of 84 mas per pixel.

Under various turbulence conditions (including telescope pointing altitude), we have recorded 322 couples of Open/Closed loop images on the Comcam at $750 \mathrm{~nm}$, and for each measured the EE in a $0.2 \operatorname{arcsec}^{2}$ box around a bright star found in the FoV. Figure 4 shows the histogram of the ratio between the Closed and Open Loop EE. It ranges from 1.0 (GLAO didn't bring any improvement) to 4.0 with a median value of 2.0 , fulfilling the requirements.

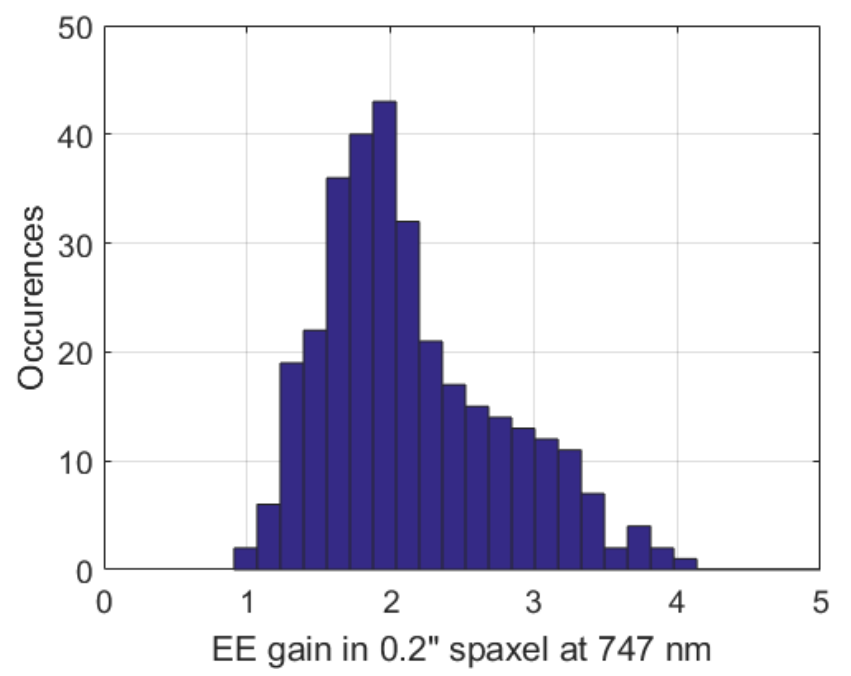

Figure 4 Histogram of gain in Ensquared Energy between Closed and Open GLAO loop with GALACSI.

This means that in average, GALACSI will allow MUSE to reach the same SNR in half the time, in addition to an increased spatial resolution. This can also be illustrated by remembering that GLAO is often considered as a "seeingenhancer" or "site-improvement" mode. The seeing or more precisely the Image Quality (IQ) can be measured as the FWHM of long-exposure images. The FWHM was measured on the same couples of images used to produce Figure 4, and its distribution plotted on Figure 5 for the Open and Closed Loop images.

The Open Loop FWHM distribution (at $750 \mathrm{~nm}$ ) has a median around 0.6 arcsec, consistent with the median Paranal seeing of $0.85 \operatorname{arcsec}$ at $500 \mathrm{~nm}$ and an Outer Scale $\mathrm{L}_{0}$ of 22 meters. The Closed Loop distribution highlights the improvement brought by GLAO: one gets a median IQ $(0.3 \operatorname{arcsec})$ which is never ever obtained without AO.

Simulations of the GALACSI WFM AO system have always shown that thanks to its design (bright LGS, high-order WFS and DSM, low latency), it performance in terms of EE gain should be most of the time limited by one factor: the ratio of turbulence present in the volume correctible by this GLAO system, i.e. approximately in the first kilometer ("Ground Layer"). 
During some of the commissioning nights, we had organized to have profiles delivered by the high-resolution StereoSCIDAR ${ }^{[6]}$ mounted on one of the VLTI $1.8 \mathrm{~m}$ diameter Auxiliary Telescopes (AT) on the Paranal platform. This instrument delivers every minute a vertical turbulence profile above the site with a resolution of $\sim 300$ meters. From those profiles, we can extract the contribution of the first kilometer above the Unit Telescopes on which GALACSI is mounted, as well as the total seeing above that telescope. The preliminary plot shown on Figure 6 shows the EE gain as function of seeing and Ground ratio of turbulence. Apart from some outliers that need to be studied, a trend is clearly visible: the EE gain performance depends little on the total seeing but is strongly correlated with the Ground layer of turbulence, as expected. An EE gain larger than 2.0 is reached when the Ground layer contribution is more than $60 \%$ of the total.

We have thus shown that the performance of GALACSI WFM in operation will not be limited by internal factors (noise, optimizations, controlled modes...) but only by the external factor of the Turbulence profile.

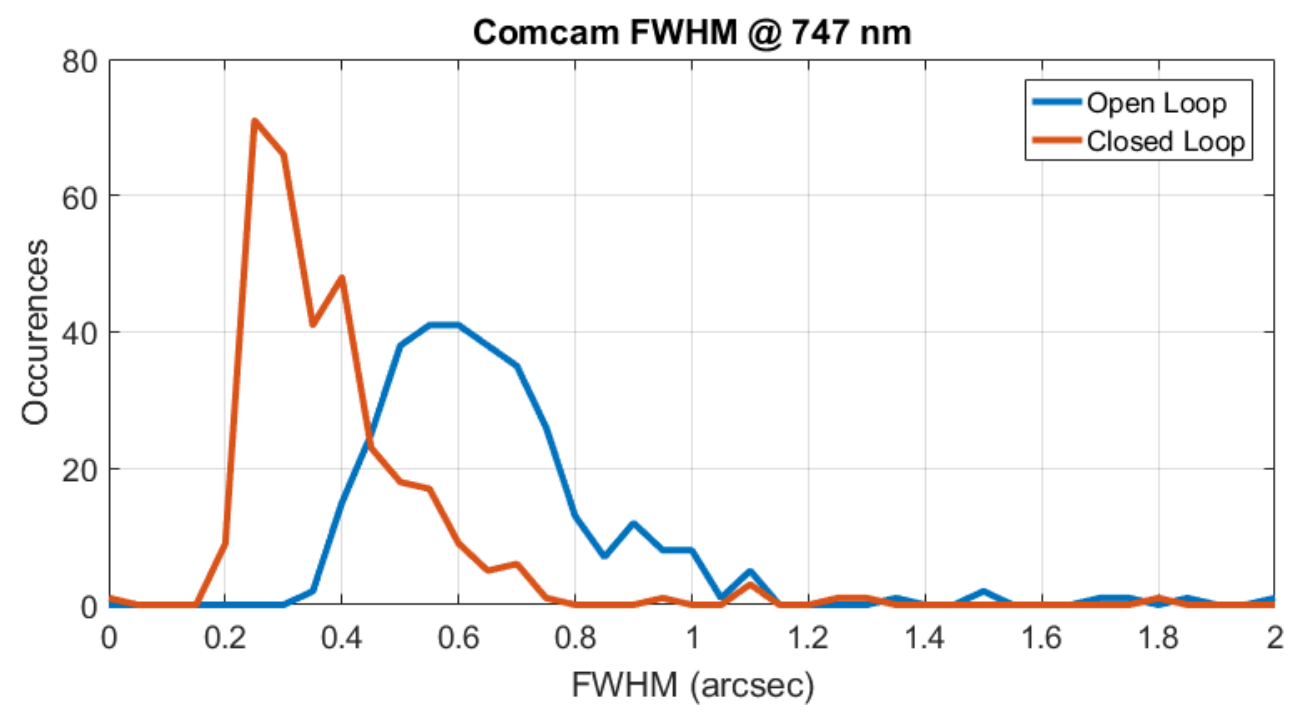

Figure 5 Distribution of Open and Closed Loop FWHM recorded on the GALACSI Comcam.

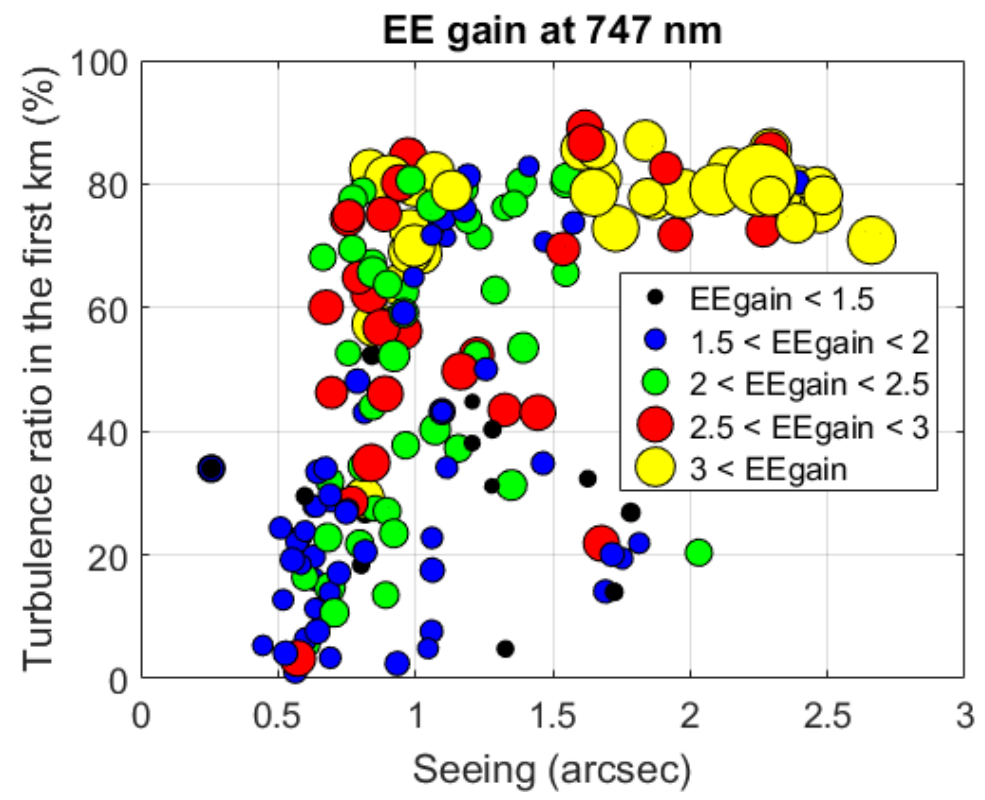

Figure 6 GALACSI WFM EE gain (bubbles size and color) measured on its Comcam as function of seeing (horizontal axis) and turbulence ratio in the first $\mathrm{km}$ of atmosphere above it (vertical axis). 


\section{REFERENCES}

[1] Pascale Hibon, "Re-commissioning of the VLT/UT4 telescope after the Deformable Secondary Mirror instalation", poster P2006, this conference

[2] Domenico Bonaccini Calia, "Laser Guide Star return flux at Cerro Paranal and at the Canary Islands", this conference

[3] Paolo La Penna, "GALACSI on the mountain - first on-sky stand-alone results", poster P1040, this conference

[4] Sylvain Oberti, "AOF laser tomography mode: reconstruction strategy and first test results", SPIE conference 2016

[5] Johann Kolb, "Laboratory results of the AOF System Testing", SPIE conference 2016

[6] James Osborn, "Stereo-SCIDAR at ESO: One year of results", poster P3019, this conference 